\title{
Die Neuregelung des Volksentscheids in Bremen vom 1. September 2009
}

\section{Dian Schefold}

1. Die bremische Landesverfassung (LV) kennt schon in der ursprünglichen Fassung $^{1}$ in einem besonderen Unterabschnitt ${ }^{2}$ eine Regelung des Volksentscheids. Vorgesehen waren drei Fälle, ein Verfassungsreferendum (Art. 70 Abs. 1 S. 1 Buchst. a), ein Referendum nach Unterbreitung einer Frage an den Volksentscheid durch die Bürgerschaft (Art. 70 Abs. 1 S. 1 Buchst. b) sowie ein Volksentscheid auf Volksbegehren (Art. 70 Abs. 1 S. 1 Buchst. c). In der Tat ist die Verfassung am 12. Oktober 1947 durch Volksentscheid - mit getrennter Abstimmung über den Mitbestimmungs-Artikel 47 - angenommen worden. ${ }^{3}$ Aber in der Folge blieben der Volksentscheid und das zu seiner Durchführung erlassene Gesetz ${ }^{4}$ fast ein halbes Jahrhundert toter Buchstabe. Die Bürgerschaft unterbreitete, abgesehen von der vom Bundesverfassungsgericht für unzulässig erklärten und daher unterbliebenen Volksbefragung zur Atombewaffnung, ${ }^{5}$ keine Frage dem Volksentscheid. Zu einem - erfolglosen - Zulassungsantrag kam es nur einmal, ${ }^{6}$ zu einem Volksbegehren überhaupt nicht, wenig verwunderlich angesichts eines Antragsquorums von einem Fünftel der Stimmberechtigten. Verfassungsänderungen blieben selten - nur sechs bis 1993 - und waren ohne Volksentscheid möglich; denn Art. 70 Abs. 1 S. 1 Buchst. a, 125 Abs. 4 LV nahmen, anders als die bayerische und die hessische Verfassung, Verfassungsänderungen bei einstimmigem Beschluss in Anwesenheit der Mehrheit der Mitglieder der Bürgerschaft vom obligatorischen Verfassungsreferendum aus. Dass dies unbe-

1 Vom 21.10.1947 (Brem.GBl. S. 251); das Datum ist das der Verkündung durch den Senat, nicht der Annahme im Volksentscheid.

2 Dritter Hauptteil, 2. Abschnitt, I. Unterabschnitt „Der Volksentscheid“, Art. 69-74, der durch Art. 125 III ergänzt wird.

3 Vgl. das Gesetz vom 9.9.1947 (Brem.GBl. S. 193).

4 Vom 1.4.1969 (Brem.GBl. S. 39) - also mehr als 20 Jahre nach Verabschiedung der Verfassung.

5 Gesetz vom 20.5.1958 (Brem.GBl. S. 49), für nichtig erklärt durch BVerfGE 8, 104.

6 Antrag auf Zulassung vom 19.6.1985, wegen Verstoßes gegen das Rechtsstaatsprinzip auf Antrag des Senats nicht zugelassen durch Entscheidung des Staatsgerichtshofs - St 2/85 - vom 9.6.1986, StGHE 4, 96. 
friedigend war, liegt auf der Hand. ${ }^{7}$ Aber das Beteiligungsquorum der Hälfte der Stimmberechtigten, im Fall von Volksbegehren sogar zum Zustimmungsquorum erhöht (Art. 72 LV), ließ den ordentlichen Weg der Verfassungsänderung durch Volksentscheid wenig verlockend erscheinen.

Hatte sich die Verfassungspraxis deshalb fast fünfzig Jahre lang mit den erwähnten einstimmig in der Bürgerschaft beschlossenen Verfassungsänderungen beholfen, so wurde die Situation kritisch, als einerseits im Zug der Verfassunggebungswelle der neunziger Jahre stärkere Anpassungen des Verfassungstexts wünschbar erschienen, andererseits die DVU Einzug in die Bürgerschaft gehalten hatte und daher in grundlegenden Fragen Einstimmigkeit nicht mehr zu erzielen war. Nun wurde erstmals, durch einen besonderen Ausschuss für Verfassungsreform, eine umfangreichere Novelle ${ }^{8}$ erarbeitet und nach - jetzt in der Tat nicht einstimmiger - Verabschiedung in der Bürgerschaft dem Volksentscheid unterbreitet. Da als Abstimmungstag der 16. Oktober 1994, das Datum der Bundestags-Wahl, vorgesehen wurde, erschien die damals noch bestehende Hürde des Beteiligungsquorums überwindbar. Dennoch, dieser erste Volksentscheid seit 1947 mochte nicht recht befriedigen. Die Vorlage änderte eine Vielzahl von Bestimmungen des organisatorischen (dritten) Hauptteils der Verfassung, ohne einen inneren Zusammenhang erkennen zu lassen. ${ }^{9}$ Teils ging es um schlichte, auch formelle Anpassungen an veränderte Umstände, namentlich des Bundesrechts, ${ }^{10}$ teils um sonstige, sachlich kaum umstrittene Modernisierungen ${ }^{11}$ unterschiedlicher Art, teils um echte Veränderungen. $\mathrm{Zu}$ diesen gehörte, neben dem Bürgerantrag, einem kaum praktikablen Volksbegehren auf Beendigung der

7 E. Kulenkampff/H. Coenen, Die Landesverfassung der Freien Hansestadt Bremen, in: JöR 3 (1954), 179 (197) berichten von einem - nicht zur Entscheidung gelangten - Antrag an den Staatsgerichtshof, der das Einstimmigkeitserfordernis zu beseitigen suchte.

8 Vgl. den Bericht des nichtständigen Ausschusses vom 3.5.1994, Bürgerschafts-Drucksache (im folgenden Bgt.-Drs.) 13/897.

9 Vgl. den - für sich schwer verständlichen - Text des Gesetzes zur Änderung der Landesverfassung der Freien Hansestadt Bremen vom 1.11.1994 (Brem.GBl. S. 289) - vier zweispaltige Seiten des Gesetzblatts mit 33 großenteils noch untergliederten Ziffern. Zur Kritik O. Jung, Die Angst der politischen Klasse vor dem Volk, in: H.H. v. Arnim (Hrsg.), Defizite in Staat und Verwaltung, 2010, S. 105 (145); ders., Die Landesverfassungsreferenden des Jahres 1994. Daten und Probleme, in: LKV 1995, 319 ff.; ders., Regieren mit dem obligatorischen Verfassungsreferendum: Wirkung, Konterstrategie, Nutzungsversuche und Umgangsweise, in: ZParl 36 (2005), 161 (169 ff.).

10 Beispiele: Regelung des Petitionsrechts (Art. 105 V n.F.), Abschaffung der Bestätigung eines Todesurteils durch den Senat (Art. 121 II a.F.).

11 Beispiele: die neuen Regelungen über Fraktionen, parlamentarische Opposition, Zusammenarbeit von Senat und Bürgerschaft bei Planungen (Art. 77-79 n.F.), Petitionsausschuss (Art. $105 \mathrm{~V}$ n.F.). 
Wahlperiode der Bürgerschaft und einer Halbierung des Antragsquorums für Volksbegehren auf Erlass eines Gesetzes, ${ }^{12}$ auch die Neuregelung der Verfassungsänderung. Dafür sollte, neben der Billigung durch Volksentscheid, nach dem neu gefassten Art. 125 Abs. 3 (in Verbindung mit Art. 70 Abs. 1 S. 1 Buchst. a) LV künftig auch eine Mehrheit von zwei Dritteln der Mitglieder der Bürgerschaft genügen; nur die für die Stellung Bremerhavens maßgeblichen Verfassungsgrundsätze sollten bei Verzicht auf den Volksentscheid nach wie vor nur durch einstimmigen Beschluss der Bürgerschaft geändert werden können. ${ }^{13}$ Worüber, wie und auf welcher Grundlage sollten angesichts dieser umfangreichen Novelle die Stimmberechtigten entscheiden? Weder ging es um eine einzelne konkrete, mit „Ja“ oder „Nein“ zu beantwortende Sachfrage, noch um ein Gesamtkonzept der Verfassung, sondern um eine eher dezisionistisch getroffene Auswahl von Änderungspunkten. Auch der - nur ganz summarisch erläuterte amtliche Abstimmungstext gab darüber kaum Aufschluß. ${ }^{14}$ So mochte das Ergebnis viele schwerlich befriedigen. Wenn seine Anfechtung vor dem Bremischen Staatsgerichtshof ${ }^{15}$ auch insgesamt ohne Erfolg blieb, deutet die Argumentation der Entscheidung doch Grenzen regierungsamtlicher Einflussnahme an.

Folge der Verfassungsreform von 1994 war nun vor allem die Öffnung für weitere Verfassungsänderungen, seit 1994 insgesamt $15,{ }^{16}$ ganz unterschiedlicher Komplexität und Tragweite, aber alle mit der erwähnten Zweidrittelmehrheit der Bürgerschaft, also ohne Volksentscheid, verabschiedet. Darunter holte schon die dritte Novelle, 1997, die 1994 unterbliebene Umwandlung des Beteili-

12 Bürgerantrag Art. 87 II, Volksbegehren auf Beendigung der Wahlperiode durch ein Fünftel der Wahlberechtigten (!), Art. 70 I 1 lit. c, 76 mit zusätzlichem und praktisch relevanterem Selbstauflösungsrecht der Bürgerschaft, während gleichzeitig dieses bisher unrealistische Antragsquorum bei Volksbegehren auf Erlass eines Gesetzes (nicht aber Verfassungsänderungen!) auf die immer noch hohe, aber überwindbare Hürde von einem Zehntel der Stimmberechtigten gesenkt wurde, Art. 70 I 1 lit. d n.F.

13 Art. 125 IV n.F., in auffälligem Kontrast zum Schutz anderer Verfassungsprinzipien, insb. Art. 20 LV. Zur Kritik A. Bovenschulte/A. Fisahn, Die Verfassung der Freien Hansestadt Bremen, in: A. Fisahn (Hrsg.), Bremer Recht, 2002, S. 18 (37 f.).

14 Vgl. das Schreiben des Statistischen Landesamts Bremen - Wahlamt - und Bremerhaven vom September 1994 an alle Wählerinnen und Wähler. Allerdings wurde es durch eine von der Bremischen Bürgerschaft herausgegebene Informationsbroschüre: Volksentscheid 1994, Bremen 1994, ergänzt.

15 E. v. 29.7.1996 - St 3/95 - NVwZ 1997, 264; zur Problematik D. Schefold, Hundertfünfzig Jahre Bremische Verfassung, in: Jahrbuch der Juristischen Gesellschaft Bremen 2000, S. 7 (19 ff.).

16 Auflistung bei D. Schefold/M. Stauch/M. Ernst (Hrsg.), Landesrecht Bremen, 14. Aufl. 2010, S. 11 f. Seither sind noch die Änderungen vom 23.3.2010 (Brem.GB1. S. 273) und vom 31.8.2010 (Brem.GB1. S. 457) nachzutragen. 
gungsquorums bei Volksentscheiden in ein Zustimmungsquorum von 25\% - bei Volksentscheiden über Volksbegehren auf Verfassungsänderung allerdings nach wie vor $50 \%$ - nach. ${ }^{17}$

2. Wichtiger war jedoch, dass das 1994 verminderte Antragsquorum für Volksentscheide, das auch in einer Neufassung des Gesetzes über das Verfahren beim Volksentscheid $^{18}$ berücksichtigt wurde, jetzt Volksbegehren auch praktisch möglich machte. Es kam, vor allem in den Jahren 1996 bis 1999, zu mehreren Anträgen auf Zulassung eines Volksbegehrens, wie sie das Gesetz ${ }^{19}$ verlangte, insofern das zweistufige Verfahren von Volksbegehren und Volksentscheid, das die Verfassung vorsah, einem dreistufigen Verfahren mit vorgeschaltetem Zulassungsverfahren annähernd. ${ }^{20}$

a) Das Ergebnis der Anträge war allerdings mager. Von den drei ersten auf Erlass von Gesetzen zur Sicherung von (1) Schulunterrichtsversorgung, (2) Schulraum und (3) Lernmittelfreiheit gerichteten Zulassungsanträgen wurden nach Einwendungen des Senats die ersten beiden durch den Bremischen Staatsgerichtshof ${ }^{21}$ als Verletzungen des Finanzvorbehalts nach Art. 70 Abs. 2 LV qualifiziert und daher für unzulässig erklärt, obwohl eine andere Argumentation im Hinblick auf Verfassungswortlaut, Verfahrensgestaltung und Vorgeschichte in der Weimarer Zeit möglich gewesen wäre. ${ }^{22}$ Maßgeblich war wohl die - angesichts der Bremer Haushaltslage - erhebliche Haushaltsbelastung. Aber in wenn auch geringerem $\mathrm{Ma}^{23}$ traf diese auch auf das dritte Vorhaben zur Sicherung der Lernmittelfreiheit zu. So blieb die Auslegung des Haushaltsvorbehalts diffus und kaum konturierte Ermessensentscheidung des Staatsgerichtshofs. Das dritte Vor-

17 Gesetz vom 14.10.1997 (Brem.GBl. S. 353), Art. 1 Nr. 6, unter Änderung des Art. 72 LV.

18 Gesetz vom 27.2.1996 (Brem.GBl. S. 41). Vgl. O. Jung, Zur Problematik des Beteiligungsquorums, in: L.P. Feld u.a. (Hrsg.), Jahrbuch für direkte Demokratie 2009, 2010, S. $<40 \mathrm{ff}$.

$19 \S 10$ des in der letzten Fn. zitierten Gesetzes, entsprechend schon seinem in Fn. 4 zitierten Vorläufer.

20 In diesem Sinn Bovenschulte/Fisahn, Verfassung (Fn. 13), S. 38; anders G. Jürgens/F. Rehmet, Direkte Demokratie in den Bundesländern - Ein Überblick, in: H.K. Heußner/O. Jung (Hrsg.), Mehr direkte Demokratie wagen, 2. Aufl. 2009, S. 197 ff. Auf die Problematik ist unten 3.b zurückzukommen.

21 E. v. 17.6.1997 - St 7/96 -, NVwZ 1998, 389 = StGHE 6, 115.

22 Vgl. die scharfe Kritik durch O. Jung, Das Finanztabu bei der Volksgesetzgebung, in: NVwZ 1998, 372 ff. m. Nachw.

23 Vgl. die Formulierung des BremStGH NVwZ 1998, 391: „nicht wesentlich“. Wo liegt die Grenze? 
haben scheiterte dann am Antragsquorum. ${ }^{24}$ - Ein weiterer Antrag auf Zulassung eines Volksbegehrens gegen die Veräußerung bremischer Anteile an der Wohnungsbaugesellschaft GEWOBA legte den Zwiespalt offen. Zwar bestätigte der auch diesmal angerufene Staatsgerichtshof seine vorerwähnte Entscheidung und erklärte auch den jetzigen Zulassungsantrag für unzulässig. ${ }^{25}$ Aber jetzt lehnten zwei Richter in einer abweichenden Meinung ${ }^{26}$ im Hinblick auf die Bedeutung der Volksgesetzgebung in der Landesverfassung, die enge Formulierung des Haushaltsvorbehalts und die verbleibenden parlamentarischen Möglichkeiten ab, den Haushaltsvorbehalt auf ein Verbot künftiger haushaltswirksamer Maßnahmen zu erstrecken. So wurden die Unsicherheit hinsichtlich des Finanzvorbehalts und das Bedürfnis nach einer Klärung weiter verdeutlicht. ${ }^{27}$

Auch die folgende Praxis trug kaum dazu bei, die Praktikabilität der Volksgesetzgebung zu stärken. Einem Antrag auf Zulassung eines Volksbegehrens gegen die Rechtschreibreform nach Schleswig-Holsteiner Muster ${ }^{28}$ gab der Staatsgerichtshof zwar überwiegend statt, aber unter Erklärung der Verfassungswidrigkeit und daher Eliminierung eines Satzes, der den zuständigen Senator zum Hinwirken auf Erhaltung der bisherigen Rechtschreibung auch über die Landesgrenzen hinaus verpflichten wollte ${ }^{29}$ - und damit unter Abschwächung des postulierten Inhalts. In der Folge kam es nicht zu einem Volksbegehren. ${ }^{30}$

b) Gravierender noch war die am gleichen Tag durch Entscheidung des Staatsgerichtshofs $^{31}$ beendete Diskussion um die Zulassung eines weiteren Volksbegehrens, in diesem Fall auf Verfassungsänderung, durch die Volksbegehren und Volksentscheid verfahrensmäßig erleichtert werden sollten. Insbesondere ging es darum, die Unterschriftenzahl für das Antragsquorum zu reduzieren und

24 Die Tabelle bei Heußner/Jung, Demokratie (Fn. 20), S. 221 (222) nennt 6,37 statt der erforderlichen 10\%. Nach der Bekanntmachung der Zulassung des Volksbegehrens vom 18.6.1997 (Brem.AB1. S. 283), wonach bis zum 25.9.1997 mindestens 50.709 Unterschriften vorliegen müssten, findet sich keine weitere offizielle Nachricht über das Schicksal des Volksbegehrens.

25 E. v. 11.5.1998 - St 3/97 -, NordÖR 1998, $297=$ StGHE 6, 180.

26 A.a.O., NordÖR 1998, 299 ff.

27 Vgl. die scharfe Kritik an der Entscheidung des BremStGH durch O. Jung, Das Finanztabu bei der Volksgesetzgebung: In Bremen nichts Neues?, in: NordÖR 1988, 281 ff.

28 Darüber hat O. Jung, „Die rebellierende Vertretung“, in: Festschrift Dian Schefold, 2001, S. 145 (146) berichtet.

29 E. v. 14.2.2000 - St 1/99 -, NordÖR 2000, $150=$ StGHE 6, 228.

30 Nach der Bekanntmachung der Zulassung des Volksbegehrens vom 6.3.2000 (Brem.ABl. S. 131) erfolgte keine weitere Bekanntmachung über das Ergebnis der Unterschriftensammlung.

31 E. v. 14.2.2000 - St 1/98 -, NordÖR 2000, $186=$ StGHE 6, 203. 
statt von der Zahl der Stimmberechtigten von der Teilnahme an der Bürgerschaftswahl abhängig zu machen, von Zustimmungs- und Beteiligungsquoren abzusehen und den Finanzvorbehalt auf „den Haushaltsplan im ganzen“ zu beschränken. Die Entscheidung des Staatsgerichtshofs operierte, ähnlich wie wenige Tage später ein Urteil des Bayerischen Verfassungsgerichtshofs, ${ }^{32}$ mit der „demokratischen Verallgemeinerungsfähigkeit“ des Volksgesetzgebungsverfahrens als Ausformung des bundesverfassungsrechtlichen Demokratieprinzips ${ }^{33}$ und erklärte deshalb die übermäßige Erleichterung der Verfassungsänderung sowie der Beendigung der Wahlperiode der Bürgerschaft, die übermäßige Erleichterung der Volksgesetzgebung für undemokratisch und die Abschwächung des Haushaltsvorbehalts für unvereinbar mit der Bindung der Bürgerschaft an das gesamtwirtschaftliche Gleichgewicht. Daher lehnte er die Zulassung des Volksbegehrens $\mathrm{ab}^{34}$

c) Nach diesen Präjudizien lag die Einschätzung nahe, die Erleichterung des Volksbegehrens durch die Verfassungsänderung von 1994 habe nicht ausgereicht, um das Verfahren operabel zu machen. Erst 2006 ergab sich eine neue Konstellation, weil ein nach Hamburger Vorbild formulierter Antrag auf Zulassung eines Volksbegehrens auf Änderung des Wahlrechts im Sinn einer stärker auf bestimmte Personen orientierten Wahl durch die Möglichkeit des Kumulierens und Panaschierens - bei Beibehaltung des durch die 5\%-Klausel nach Art. 75 Abs. 3 LV vorausgesetzten, wenn auch nicht ausdrücklich in der Verfassung vorgeschriebenen Verhältniswahlsystems - bei der Vorprüfung durch den Senat nicht abgelehnt wurde. ${ }^{35}$ Das damit zugelassene Volksbegehren hatte gleich den Erfolg, dass es von $13,5 \%$ der Stimmberechtigten unterzeichnet wurde. ${ }^{36}$ Ein Obsiegen im Volksentscheid war nicht unwahrscheinlich. Die Bürgerschaft entschloss sich in dieser Lage, das Volksbegehren unverändert als Gesetz anzuneh-

32 Vom 31.3.2000, BayVB1. 2000, 397 = BayVerfGHE 53, 42.

33 Art. 28 I 1 GG; der Bayerische Verfassungsgerichtshof rekurrierte seinerseits auf die Verankerung des Prinzips in der Bayerischen Verfassung.

34 Kritik an der Entscheidung bei O. Jung, Direkte Demokratie nach Schweizer Art in Deutschland verfassungswidrig? $\mathrm{Zu}$ einer Grundgesetzauslegung des Staatsgerichtshofs der Freien Hansestadt Bremen, in: KritV 84 (2001), 24 ff.; G. Stuby, Hut ab vor dem Volk, aber Quoren müssen her!, in: Festschrift Dian Schefold (Fn. 28), S. 243 ff.; Bovenschulte/Fisahn, Verfassung (Fn. 13), S. $41 \mathrm{f}$.

35 Zulassung durch Bekanntmachung vom 4.7.2006 (Brem.ABl. S. 469) mit Eintragungsfrist bis 18.10.2006 und mindestens 48.175 notwendigen Unterschriften.

36 So die Angaben bei Heußner/Jung, Demokratie (Fn. 20), S. 223; vgl. Bekanntmachung vom 5.12.2006 (Brem.ABl. S. 819). 
men, und entsprechend wurde das Bremische Wahlgesetz geändert. ${ }^{37}$ Daher kam es auch in diesem Fall zu keinem Volksentscheid.

3. Trotz dieses Erfolges eines Volksbegehrens zeigt die dargestellte Vorgeschichte, dass die Regelung von Volksentscheid und Volksbegehren in der Tat gravierende, noch nicht gelöste Probleme aufwarf. Den Anstoß, diese zu überdenken, gaben die Bürgerschaftswahl 2007 und die anschließende Bildung einer Koalition aus SPD und Bündnis 90/Die Grünen. Die Koalitionsvereinbarung vom 17. Juni $2007^{38}$ legte denn auch die Grundlinien der geplanten Reform fest und sah die Bildung eines entsprechenden Parlamentsausschusses vor. In der Tat setzte die Bürgerschaft bereits mit Beschluss vom 18. Oktober 2007 einen nichtständigen Ausschuss „Erleichterung der Volksgesetzgebung und Weiterentwicklung des Wahlrechts ${ }^{\text {‘39 }}$ ein, der die beiden Komplexe überdenken und zu Neuregelungen führen sollte.

a) Allerdings fällt schon an der Planung dieser Neuregelung auf, dass sie ein Problem ausklammert, das nach der hier referierten Vorgeschichte essentiell und wahrscheinlich mit kausal für die entstandenen Schwierigkeiten der praktischen Handhabung der Volksgesetzgebung war. Wie ausgeführt, hatte ja die bremische Verfassung, insofern abweichend von den aus dem gleichen verfassungspolitischen Umfeld - der damaligen amerikanischen Besatzungszone - stammenden Verfassungen Bayerns (Art. 75 Abs. 2 S. 2) und Hessens (Art. 123 Abs. 2), das obligatorische Verfassungsreferendum durch die Möglichkeit einstimmiger Beschlussfassung in der Bürgerschaft durchlöchert, und diese Durchlöcherung hat die bremische Verfassungspraxis bestimmt. Sie ist 1994 durch die Möglichkeit der Verfassungsänderung mit parlamentarischer Zweidrittelmehrheit noch erweitert und entsprechend genutzt worden. Damit fehlt es an einer Praxis der Volksentscheide, die die Stimmberechtigten in das Rechtsetzungsverfahren einbezieht. Die Neuregelung ändert hieran nichts. Zwar bleibt ein Volksentscheid wie bisher weiterhin auf Beschluss der Bürgerschaft möglich. ${ }^{40}$ Aber nichts spricht dafür, dass diese Regelung in Zukunft praktisch relevanter wird als in den letzten sechzig Jahren.

37 Gesetz zur Änderung des Wahlgesetzes vom 19.12.2006 (Brem.GBl. S. 539).

38 SPD, Landesorganisation Bremen/Bündnis 90/Die Grünen, Landesverband Bremen, Vereinbarung zur Zusammenarbeit in einer Regierungskoalition für die 17. Wahlperiode der Bremischen Bürgerschaft 2007-2011, 17.6.2007, S. 94 (96).

39 Die erste Phase von dessen Arbeit ist dokumentiert in der Bgt.-Drs. 17/594 vom 4.11.2008; danach die folgenden Hinweise.

40 Art. 70 I 1 litt. a, b LV, die unverändert geblieben sind. 
b) Im Mittelpunkt der Beratung und Neuregelung standen daher das Volksbegehren und, soweit es dazu führt, der dadurch bewirkte Volksentscheid. Insofern stellte sich das grundsätzliche Problem des zwei- oder dreistufigen Verfahrens. ${ }^{41}$ Es wird dadurch beeinflusst, dass der 1994 eingeführte Bürgerantrag ${ }^{42}$ als erste Stufe in Betracht kam, obwohl hier schon sechzehnjährige und auch ausländische Einwohner mitwirkungsberechtigt sind. Daher kann der Bürgerantrag auch als an die Bürgerschaft gerichteter Antrag auf Zulassung eines Volksbegehrens gedeutet und in einen solchen Antrag einbezogen werden - allerdings nur, wenn er die Anforderungen auch eines solchen Antrags erfüllt, sowie namentlich unter Ausklammerung der nicht stimmberechtigten Unterzeichner des Bürgerantrags. ${ }^{43}$ Die Verbindung des (unverbindlichen) Antrags an die Bürgerschaft und des (ein verpflichtendes Verfahren einleitenden) Antrags auf Zulassung eines Volksbegehrens an den Senat bleibt daher äußerlich und unvollkommen. Es ist kaum zu erwarten, dass sie dem Bürgerantrag neue Bedeutung einbringen wird.

Umgekehrt: Den Schritt, Anträge auf Zulassung eines Volksbegehrens zwingend der Bürgerschaft zu unterbreiten, hat die Neuregelung nicht tun wollen; der Antrag richtet sich nach wie vor an den Landeswahlleiter und den Senat, führt zur rechtlichen Überprüfung des Volksbegehrens und, im Fall der Zulassung, zum Eintragungsverfahren ( $\$ 12$ des Gesetzes). Erst wenn dieses zum Zustandekommen des Volksbegehrens geführt hat, wird die Bürgerschaft offiziell damit befasst. Diese Lösung - man wird sie als zweistufige einordnen - ist nach wie vor in der Verfassung verankert und im Ausführungsgesetz ausgestaltet; ${ }^{44}$ offenbar ist sie im Ausschuss nicht grundsätzlich in Frage gestellt worden. Allerdings schließt sie nicht aus, dass die Bürgerschaft ihrerseits ein Gesetz entsprechend dem Inhalt eines beantragten Volksbegehrens beschließt und damit dieses gegenstandslos macht, oder eine Alternativregelung zum beantragten Volksbegehren beschließt; dann richtet sich ein dennoch durchgeführtes Volksbegehren gegen

41 Dazu schon oben 2. Zu dieser Unterscheidung Jürgens/Rehmet, Überblick (Fn. 20), S. $197 \mathrm{ff}$.

42 Art. 87 II LV, konkretisiert durch Gesetz vom 20.12.1994 (Brem.GB1. S. 325) mit seitherigen Änderungen, zuletzt durch Art. 3 des hier behandelten Gesetzes vom 1.9.2009 (Brem.GB1. S. 311).

43 Vgl. § 10 II VolksentscheidG und dazu Bgt.-Drs. 17/594, S. 9, $12,14$.

44 Art. 70 I 1 lit. d, 3 LV, ausgeführt durch $\S \S 12-21$ VolksentscheidG, sachlich durch die Reform nicht verändert und in Bgt.-Drs. 17/594, die S. 1 das Verfahren als zweistufig qualifiziert, nicht weiter begründet. 
dieses Gesetz. ${ }^{45}$ Trotz der zweistufigen Ausgestaltung des Verfahrens zeigen die erwähnten Besonderheiten, dass es sich der dreistufigen Lösung annähert.

c) Im Einzelnen ist hervorzuheben, dass die Neuregelung den Initiatoren eines Volksbegehrens unentgeltliche Beratung durch Bürgerschaft und Senat hinsichtlich verfassungs-, haushalts- und verfahrensrechtlicher Fragen gewährleistet. ${ }^{46}$ Wichtig ist dies schon wegen der beträchtlichen Anforderungen, einen ausgearbeiteten Gesetzesentwurf vorzulegen, zumal dieser jetzt nach der Neuregelung zwingend eine Begründung enthalten muss. ${ }^{47}$

Mindestens ebenso kritisch ist sodann die Bestimmung der Tragweite des $\mathrm{Fi}$ nanzvorbehalts, der im neuen, ausführlichen Art. 70 Abs. 2 LV und dem ihn konkretisierenden $\S 9$ S. 1 Nr. 1 und S. 2 VolksentscheidG präzisiert wird. Dabei fällt vorweg auf, dass auch nach dem geänderten Verfassungstext der ,Volksentscheid“" (nicht etwa das Volksbegehren) dem Finanzvorbehalt unterliegt. Dennoch knüpft das Gesetz über den Volksentscheid auch in seiner neuen Fassung (§ 9 S. 1 Nr. 1 und S. 2) schon die Zulässigkeit des Antrags auf Zulassung eines Volksbegehrens an die Einhaltung des Finanzvorbehalts. Diese zumindest dem Verfassungswortlaut widersprechende, argumentativ mit eingehender Begründung in Frage gestellte Erstreckung ${ }^{48}$ kann sich zwar auf die Entscheidung des Staatsgerichtshofs vom 17. Juni 1997, ${ }^{49}$ speziell auf die Parallele zur Regelung des Bürgerantrags nach Art. 87 Abs. 2 Satz 2 LV, berufen. Sie wurde auch von der Bürgerschaft bei der Änderung der Landesverfassung und des Gesetzes über den Volksentscheid gewollt, da beide gemeinsam erarbeitet worden sind. Aber abgesehen von der zum gegenteiligen Ergebnis führenden grammatikalischen Interpretation schießt die Regelung auch insoweit über das Ziel hinaus, als sie neben Anträgen auf Zulassung eines Volksbegehrens und Volksentscheiden nach erfolgreichem Volksbegehren auch Volksentscheide nach Unterbreitung durch die Bürgerschaft (Art. 70 Abs. 1 S. 1 Buchst. a, b LV) dem Finanzvorbehalt

45 So ausdrücklich $\S 7$ II VolksentscheidG, der insofern die Befugnis der Bürgerschaft zum vorgezogenen Erlass einer Alternativregelung ausdrücklich erwähnt.

46 So der neue $\S 8$ a VolksentscheidG, der allerdings die Frage eines Vertrauensschutzes für dadurch bewirkte Formulierungen nicht anspricht und angesichts der Zuständigkeit des Staatsgerichtshofs wohl auch nicht präjudizieren kann. Eine Regelung hinsichtlich einer Kostenerstattung, wie sie in mehreren anderen Ländern vorgesehen ist (Jürgens/Rehmet, Überblick [Fn. 20], S. 207 ff.), wird auch durch die neue Bremer Gesetzgebung nicht getroffen.

$47 \S 10$ II Nr. 1 VolksentscheidG, dazu Bgt.-Drs. 17/594, S. 12.

48 Dazu eingehend Jung, Finanztabu (Fn. 22), S. 372 f., unter Hinweis auf die ausführlichere Darlegung durch dens., Das Finanztabu bei der Volksgesetzgebung. Die Staatsrechtslehre und Staatspraxis der Weimarer Zeit, in: Der Staat 38 (1999), $41 \mathrm{ff}$.

49 E. v. 17.6.1997 - St 7/96 -, NVwZ 1998, 388 (389). 
unterwirft. Insofern ist der Wortlaut eindeutig; auch die Bürgerschaft darf keine vom Finanzvorbehalt erfassten Materien dem Volksentscheid unterbreiten. Aber es bleibt doch die Frage, ob diese Konsequenz bedacht worden ist. Soll wirklich selbst die Bürgerschaft in finanziell bedeutsamen Fragen das Volk nicht befragen dürfen? Mit dem Schutz der öffentlichen Finanzen vor verschwendungssüchtigen Minderheiten, die bei Fehlen eines Finanzvorbehalts ein Volksbegehren starten könnten, hat dies nichts zu tun. Wohl aber wird damit ausgeschlossen, dass Sparsamkeit als Tugend der Bürgerinnen und Bürger für politische Entscheidungen nutzbar gemacht, konkret: in der prekären bremischen Haushaltssituation ein notwendiger harter Einschnitt an die Billigung in einem Volksentscheid geknüpft wird. Vor lauter Angst vor ausgabefreudigem Gebaren des Volkes scheint dieser Sinn des Volksentscheids völlig aus dem Blick geraten zu sein. Gerade in Bremen gibt es Grund genug, daran zu erinnern ${ }^{50}$ und die getroffene Lösung insofern zu kritisieren.

Freilich, die Tragweite des Finanzvorbehalts ist, nach offenbar sehr kontroversen Diskussionen, ${ }^{51}$ abgemildert worden. Der Kreis der ausgeschlossenen Materien - laufender Haushaltsplan, Bezüge oder Entgelte öffentlicher Bediensteter oder vergleichbarer Personen, Steuern, Abgaben, Beiträge und Gebühren sowie über Einzelheiten solcher Gesetzesvorlagen ${ }^{52}$ - ist zwar sachlich gleich geblieben, ja durch Präzisierung eher erweitert worden: neben dem - nahe liegenden Verbot von Eingriffen in den laufenden Haushalt umfasst er etwa auch Abgeordneten-Diäten, Bezüge Bediensteter öffentlicher Unternehmen, politisch heikle

50 Ein journalistisch ausgewiesener Beobachter, der Korrespondent der Neuen Zürcher Zeitung, U. Schmid, resümierte kürzlich unter dem Titel: „Die Lust an der politischen Schweinegrippe“ (NZZ Nr. 223 v. 25.9.2010, S. 57): „Handlungsfähigkeit ist nicht das Privileg autoritärer Eliten. Nicht die Parteiendemokratie Deutschland, nicht das zentralistische Frankreich hat die Schulden gering gehalten, sondern die Schweiz, in der der angeblich so unzuverlässige, verschwendungssüchtige und leicht zu verführende Bürger überall dreinreden kann. Das sollte nicht zu Überheblichkeit verleiten. Aber es deutet an, dass sich etwas mehr Respekt vor dem Bürger lohnen kann. Zurückhaltende staatspolitische Vernunft kommt nicht von oben, sondern von unten. Sie wächst aus den kleinen Gruppen, aus den Familien und Gemeinden, sie wächst dort, wo die Bürger den Überblick behalten haben, wo sie zuständig sind, wo sie keine Experten benötigen, die ihnen das Denken abnehmen, wo sie selber entscheiden. Man wünschte den lauten, selbstherrlichen und von ihrer Wichtigkeit so überaus überzeugten deutschen Eliten den Mut, so etwas zur Kenntnis zu nehmen.“ - Der Stadtstaat, und gerade der finanziell gebeutelte, wäre der Ort, hier den Anfang zu machen. Eingehender zur dahinter stehenden Grundsatzfrage Jung, Angst (Fn. 9), S. 105 ff.

51 Vgl. die stark abweichenden und unter sich gegensätzlichen Minderheitsvoten der Oppositionsfraktionen, Bgt.-Drs. 17/594, S. 14.

52 Art. 70 II 1 LV und gleichlautend $§ 9$ S. 1 Nr. 1 VolksentscheidG. 
Entgelte etwa der Abfallentsorgung, so dass der Bremer Finanzvorbehalt immer noch weit gefasst ist, von einer Bürgerkontrolle über das Ausgabenverhalten der Behörden kaum gesprochen werden kann. ${ }^{53}$ Aber hinsichtlich der für die Zukunft haushaltswirksamen Volksbegehren sieht die Neuregelung ${ }^{54}$ vor, dass sie zulässig sind, wenn sie „,die Struktur eines zukünftigen Haushalts nicht wesentlich verändern, den verfassungsrechtlichen Regelungen des Haushaltsrechts, welchen auch die Bürgerschaft für die Aufstellung des Haushaltsplans unterliegt, entsprechen und zur Gegenfinanzierung keine Haushaltspositionen herangezogen werden, die gesetzlich, vertraglich oder auf andere Weise rechtlich gebunden sind“. Zusätzlich wird ein Finanzierungsvorschlag gefordert. Es fällt leicht, in dieser Regelung einen Anklang an das Minderheitsvotum zweier Richter des Staatsgerichtshofs zur Nichtzulassung des Volksbegehrens gegen Veräußerung von Anteilen der GEWOBA ${ }^{55}$ zu finden. Aber dieses Präjudiz zeigt auch, wie schwierig eine Volksgesetzgebung ist, die die finanziellen Rahmenbedingungen respektiert und Prioritäten verändern will - vor allem wenn auch die vom Volksentscheid ausgeschlossenen Materien unangetastet bleiben müssen. In diesem Korsett Volksbegehren zu formulieren, wird angesichts der bestehenden Haushaltsprobleme nach wie vor sehr schwierig sein. So begrüßenswert daher das Signal der Neuregelung ist, so wenig unmittelbarer Erfolg darf erwartet werden. Realistischer wäre, die Bürgerbeteiligung zugunsten der Sparzwänge zu nutzen. Aber dem steht nach wie vor der Finanzvorbehalt entgegen.

d) Die übrigen Voraussetzungen für Anträge auf Zulassung von Volksbegehren und deren Prüfung sind nur geringfügig oder gar nicht modifiziert worden. Nach wie vor braucht der Antrag auf Landesebene 5000, in der Stadtgemeinde Bremen $^{56} 4000$ Unterschriften, was eher im oberen Bereich der Anforderungen in den Ländern liegt, aber keine allzu hohe Hürde aufrichtet. ${ }^{57}$ Schon im Hinblick auf die Anträge ist wichtig, dass die Rolle der Vertrauenspersonen ( $§ 10$

53 Dazu die vergleichende Übersicht bei $F$. Wittreck, Direkte Demokratie vor Gericht, in: Heußner/Jung, Demokratie (Fn. 20), S. 397 (413 f.); ausführlicher J. Krafczyk, Der parlamentarische Finanzvorbehalt bei der Volksgesetzgebung, 2005, S. 25 ff., dessen Einebnung der unterschiedlichen Formulierungen hier freilich nicht geteilt wird.

54 Art. 70 II 2 (Zitat im Text), Art. 71 II LV, wiederholt in $\S 9$ S. 2, § 10 II Nr. 1 VolksentscheidG; dazu Bgt.-Drs. 17/594, S. 9 f., 12.

55 BremStGH, E. v. 11.5.1998 - St 3/97 -, NordÖR 1998, 297 (299 ff.), s.o. bei Fn. 25 ff.

56 Zur kommunalen Volksgesetzgebung M. Göbel, Bremen, in: T. Mann/G. Püttner (Hrsg.), Handbuch der kommunalen Wissenschaft und Praxis, Bd. 1, 3. Aufl. 2007, S. 771 (790 f., 795).

57 Vgl. die Synopse bei Jürgens/Rehmet, Überblick (Fn. 20), S. 198 f. 
Abs. 2 Nr. 3 VolksentscheidG) durch die Neuregelung an Gewicht gewonnen hat, wie sogleich zu zeigen sein wird.

e) Wird dem Zulassungsantrag, gegebenenfalls nach Entscheidung des Staatsgerichtshofs, stattgegeben, so beginnt die dreimonatige Eintragungsfrist. Dies und die Zulässigkeit freier Unterschriftensammlung entsprechen bisherigem Recht. Davon abweichend wird jedoch - und das ist einer der Hauptpunkte der Reform, auf den großes Gewicht gelegt wurde ${ }^{58}$ - das Unterschriftenquorum für Volksbegehren auf Gesetzesstufe von zehn auf fünf Prozent der Stimmberechtigten abgesenkt, Art. 70 Abs. 1 S. 1 Buchst. d LV, § 19 Abs. 2 VolksentscheidG. Dieser Wert macht nun in der Tat das Volksbegehren zu einem nutzbaren Instrument. Aber eben nur für Gesetze! Für Volksbegehren auf Verfassungsänderung bleibt das bisherige 20\%-Quorum bestehen; darauf insistierte die SPD. ${ }^{59}$ Der Grund, vielleicht zu suchen im Konflikt um den Antrag auf Verfassungsänderung von $2000,{ }^{60}$ ist schwer verständlich. Da auch Landesverfassungsänderungen den Vorrang des Bundesrechts wahren müssen, sind rechtsstaats- oder menschenrechtswidrige Volksbegehren auch auf Verfassungsstufe, die besondere Vorsicht gegenüber solchen Begehren rechtfertigen mögen, kaum denkbar. Es mag dennoch sinnvoll sein, die Latte für solche Begehren zur Wahrung des Vorrangs der Verfassung etwas höher zu legen. Aber ein vierfaches Antragsquorum, noch dazu verbunden mit einem prohibitiven Zustimmungsquorum, scheint mir denn doch über das Ziel hinaus zu schießen.

f) Differenzierter sind durch die Reform in Art. 70 Abs. 1 S. 1 Buchst. d, S. 3$5 \mathrm{LV}$, ausgeführt in $\S 21$ VolksentscheidG, die Modalitäten der Behandlung erfolgreicher Volksbegehren geregelt. Hier, im insofern eindeutig zweistufigen Verfahren, wird auf Vorlage des Senats die Bürgerschaft eingeschaltet. Dabei sind vier Fälle zu unterscheiden.

(1) Gibt die Bürgerschaft dem Volksbegehren nicht innerhalb von vier Monaten statt, so müssen die Vertrauenspersonen innerhalb der Ausschlussfrist (!) eines weiteren Monats den Antrag auf Durchführung des Volksentscheids stellen. Diese neue Anforderung ${ }^{61}$ mag der Rechtssicherheit dienen, erlegt aber den Ver-

58 Vgl. schon die oben Fn. 38 zitierte Koalitionsvereinbarung vom 17.6.2007, S. 96, sodann Bgt.-Drs. 17/594, S. $2,9$.

59 Bgt.-Drs. 17/594, S. 9 in Verbindung mit den S. 14 wiedergegebenen Minderheitsmeinungen. Die Standpunkte wurden in der parlamentarischen Beratung nochmals verdeutlicht, Bremische Bürgerschaft, Plenar-Protokoll 17/38 vom 19.2.2009, S. 2929 f.; 17/46 vom 28.5.2009, S. 3361 f., 3366 f.; $17 / 50$ vom 27.8.2009, S. 3713 ff.

60 Vgl. BremStGH E. 14.2.2000 - St 1/98 -, NordÖR 2000, 186, dazu oben bei Fn. 31.

61 Art. 70 I 1 lit. d, 4 LV, $\S \S 1$ Nr. 4, 21 IV VolksentscheidG. Bgt.-Drs. 17/594, S. 9, 13 stellt die Anforderung einfach fest, ohne sie zu begründen. 
trauenspersonen eine Verantwortung und Verfügungsbefugnis auf, die das Legitimationsproblem aufwirft - die Bestellung der Vertrauenspersonen vollzieht sich ja völlig im außerstaatlichen Bereich - und zu praktischen Konflikten führen kann.

(2) Diese können sich zuspitzen, wenn die Bürgerschaft dem Volksbegehren stattgeben will. Der Normalfall - unveränderte Übernahme des Volksbegehrens und Erlass eines entsprechenden Gesetzes - mag noch eindeutig sein; aber schon hier kann die Bürgerschaft längere Entscheidungszeit beanspruchen. Für diesen Fall sieht jetzt der neue $\S 21$ Abs. 5 eine bis zu zweimonatige Verlängerung der Entscheidungsfrist vor, aber nur auf Antrag der Vertrauensleute. Diese haben also die Wahl, ob sie sich auf dieses Verfahren einlassen oder die Ansetzung des Volksentscheids beantragen wollen; dieser hat dann vorbehaltlich weiterer Ausnahmemöglichkeiten innerhalb weiterer vier Monate stattzufinden ( $\$ 2$ VolksentscheidG).

(3) Noch delikater wird die Situation, wenn die Bürgerschaft dem Volksbegehren in veränderter, aber seinem Anliegen grundsätzlich entsprechender Form stattgeben will. Diese Konstellation, häufig und unvermeidlich bei einem neuen Gesetz, das oft nicht alle Folgewirkungen, z.B. auf andere Gesetze, einbeziehen wird, ${ }^{62}$ kann zu Veränderungen des im Volksbegehren beantragten Texts führen. Dies können durchaus im Sinn des Begehrens sein, möglicherweise sogar seinem Anliegen besser entsprechen; aber ebenso gut möglich ist, dass sie das Begehren in seinem Inhalt verändern, verfälschen oder seiner Bedeutung berauben. Die Neuregelung (§ 21 Abs. 3) legt nun die Entscheidung auch darüber durch Antrag auf Erledigung des Volksbegehrens in den Verantwortungsbereich der Vertrauenspersonen. Die Praxis wird zeigen müssen, ob diese dadurch nicht überfordert, ob nicht Konflikte um den Zweck eines Volksbegehrens heraufbeschworen werden.

(4) Klassische Situation des Parlaments gegenüber einem Volksbegehren ist demgegenüber, dass wohl die Regelungsbedürftigkeit der Materie anerkannt, aber eine andere Lösung bevorzugt und deshalb als Gegenvorschlag formuliert wird. § 21 VolksentscheidG regelt diesen Fall nicht ausdrücklich und lässt offen, ob ein Gegenvorschlag als Gesetz erlassen und unter Berufung darauf das

62 Typisch der insofern offenbar eindrückliche Beispielsfall des Volksbegehrens zum Wahlrecht, dem die Bürgerschaft mit dem Gesetz vom 19.12.2006 (dazu oben bei Fn. 37) stattgegeben hatte - und das in der Folge zu zahlreichen Anpassungen, vor allem des Wahlgesetzes, führte, von denen die meisten den Intentionen des Volksbegehrens nicht widersprachen, andere aber möglicher- (und umstrittener-)weise den Kern des Volksbegehrens trafen, dazu unten 4. 
Volksbegehren zur Ablehnung empfohlen wird - was die Folge hätte, dass es im Fall des Erfolgs an die Stelle des parlamentarischen Gesetzes träte ${ }^{63}-$, oder ob der Gegenvorschlag als Vorlage der Bürgerschaft im Sinn des Art. 70 Abs. 1 S. 1 Buchst. b LV mit dem Volksbegehren zur Abstimmung gebracht werden soll. In der Regelung des Volksentscheids ${ }^{64}$ wird dafür jedoch ein passendes Verfahren mit mehreren konkurrierenden Vorlagen und einer Stichfrage im Fall der Annahme mehrerer Vorlagen entwickelt: Gesetz wird die in der Stichfrage bevorzugte Vorlage. Die Regelung zeigt, dass auch der Bremer Gesetzgebung der Gegenvorschlag durchaus bekannt ist, ${ }^{65}$ wenn es auch dazu, wie zum Volksentscheid überhaupt, eben noch keine Praxis gibt.

g) Insgesamt zeigen auch diese sehr differenzierten, aber, wie gezeigt, konfliktträchtigen Regelungen eine Tendenz, trotz Erleichterung der Volksbegehren, namentlich durch die Senkung des Quorums, Volksentscheide möglichst zu vermeiden. Kommt es dennoch dazu, ist vor allem eine bedeutsame Neuerung hervorzuheben: Auch das Zustimmungsquorum zu Gesetzen wird von bisher 25 auf $20 \%$ der Stimmberechtigten abgesenkt, Art. 72 Abs. 1 LV, § 6 Abs. 1 S. 2 VolksentscheidG. Das erhöht, vor allem im Vergleich zum früheren Beteiligungsquorum von $50 \%$, die Chancen von Volksbegehren beträchtlich. Für Verfassungsänderungen (und auch Volksentscheide über die Auflösung der Bürgerschaft) dagegen ist, trotz heftiger Kontroverse, ${ }^{66}$ das Zustimmungsquorum von $50 \%$ der Stimmberechtigten beibehalten worden, Art. 72 Abs. 2 LV. Zusammen mit dem erwähnten, für sich schon schier prohibitiven Antragsquorum von 20\% dürfte dies gewährleisten, dass es in Bremen auch künftig weder Verfassungsänderungen noch Beendigungen der Wahlperiode durch Volksentscheid geben wird.

Auffallen muss außerdem, dass das Zustimmungsquorum von 20\% gemäß dem eindeutigen Verfassungswortlaut nach wie vor für alle Vorlagen nach Art. $70 \mathrm{LV}$, also auch die der Bürgerschaft gilt. Das mag für diese den Anreiz, eine „,zu ihrer Zuständigkeit gehörende Frage dem Volksentscheid“ (Art. 70 Abs. 1 S. 1 Buchst. b LV) zu unterbreiten, und damit die Häufigkeit von Volksentscheiden weiter vermindern. Im Übrigen beschränken sich die Änderungen der Regelung des Volksentscheids auf technische Details.

63 So ausdrücklich $\S 7$ II VolksentscheidG.

$64 \S 2$ II $3, \S 3$ insb. IV, § 6 VolksentscheidG.

65 Dies gilt im Prinzip schon seit dem Gesetz i.d.F. vom 27.2.1996 (Brem.GBl. S. 41); insofern ist die Aussage bei Jürgens/Rehmet, Überblick (Fn. 20), S. 205 zu korrigieren.

66 Vgl. Bgt.-Drs. 17/594, S. 14, die Absenkungsbestrebungen auch von CDU, FDP und Linkspartei vermerkt; erst recht wurden diese von den Grünen unterstützt, prallten aber an der Position der SPD ab. 
Zusammengefasst lässt sich sagen, dass die Versprechen der Koalitionsvereinbarung, bei Gesetzen das Antragsquorum auf fünf Prozent, das Zustimmungsquorum auf $20 \%$ zu reduzieren und den Finanzvorbehalt enger zu fassen, eingehalten worden sind. Für Verfassungsänderungen ist die Regelung dagegen nicht verbessert worden. Die Änderung des Finanzvorbehalts dürfte dessen Probleme kaum lösen, und die komplizierte, konfliktträchtige parlamentarische Behandlung von Volksbegehren erscheint vor allem von dem Bestreben geprägt, Volksentscheide möglichst zu vermeiden. Dies wird auch an der Regelung der Kontralegislatur deutlich, auf deren Problematik im Folgenden anhand des konkreten Beispiels einzugehen ist.

4. Die Bedeutung der Neuregelung der Volksgesetzgebung wird eher verständlich, zieht man den konkreten Ausgangspunkt des Volksbegehrens auf Änderung des Wahlrechts in Betracht, dem die Bürgerschaft, wie ausgeführt, noch am Ende der letzten Wahlperiode und ohne Volksentscheid stattgegeben hat - freilich in einer sehr komplizierten, technischen Materie, und nur mit Wirkung von der übernächsten Wahl (2011) an. In der Tat war die Zustimmung zur Neuregelung nicht völlig vorbehaltlos, die Zeitspanne bis zur erstmaligen Anwendung des neuen Gesetzes lang und das Bedürfnis nach Harmonisierung zwischen dem erfolgreichen Volksbegehren und den Einzelheiten der intrikaten Materie offenkundig. Die Koalitionsvereinbarung ließ dies bereits durchblicken, ${ }^{67}$ und der Ausschuss der Bürgerschaft, der die Neuregelung der Volksgesetzgebung vorbereiten sollte, erhielt daher von Anfang an den Auftrag, sich auch mit der Weiterentwicklung des Wahlrechts zu befassen. Im Einsetzungsbeschluss ${ }^{68}$ war außer von Wahlrecht für 16- und 17-Jährige, Ausländerwahlrecht und Wahlrecht für die Beiräte auch von ,als wünschenswert erachteten Anpassungen im Landeswahlrecht" die Rede. Drohte hier Kontralegislatur?

a) Die Frage wurde verblüffend schnell konkret. Alsbald nach Einsetzung des nichtständigen Ausschusses forderte die Stadtverordnetenversammlung Bremerhaven die Wiedereinführung der 5\%-Klausel für die dortigen Wahlen, und die Bremer Koalitionsfraktionen beeilten sich, die Verwirklichung dieses Postulats durch Dringlichkeitsantrag auf Änderung des kürzlich gemäß dem Volksbegeh-

67 Vgl. die oben Fn. 38 erwähnte Koalitionsvereinbarung, S. 95, die speziell, wenn auch verklausuliert, die Wiedereinführung der durch das Volksbegehren abgeschafften 5\%Klausel in Bremerhaven erwähnte.

68 Bgt.-Drs. 17/88 vom 18.10.2007, Ziff. 1-5 (6 und 7 betrafen die Volksgesetzgebung). 
ren erlassenen Änderungsgesetzes zum Wahlgesetz zu fordern. ${ }^{69}$ Allerdings erwies sich die Frage schon im Ausschuss als nicht unproblematisch, weder politisch, noch rechtlich, zumal das Bundesverfassungsgericht soeben die Beibehaltung der 5\%-Klausel im Kommunalwahlrecht Schleswig-Holsteins für verfassungswidrig erklärt hatte. ${ }^{70}$ Daher verständigten sich der Ausschuss und, ihm folgend, die Bürgerschaft dahin, zunächst die verfassungsrechtliche Zulässigkeit der Wiedereinführung der 5\%-Klausel in Bremerhaven durch den Staatsgerichtshof prüfen zu lassen. ${ }^{71}$ Erst nach dieser Klärung sollte die politische Diskussion über die Wiedereinführung geführt werden - wobei der diesbezügliche Antrag der Koalition bereits auf dem Tisch lag und durch eine positive Entscheidung des Staatsgerichtshofs wohl entscheidenden Rückenwind bekommen hätte. Einen solchen Wind ließ das Urteil ${ }^{72}$ insofern spüren, als es die Änderung eines auf Grund Volksbegehrens parlamentarisch beschlossenen Gesetzes für verfassungsrechtlich, speziell im Hinblick auf das Demokratieprinzip, unbedenklich und die Grenzen der Kontralegislatur für nicht betroffen erklärte. Zur kommunalrechtlichen Sperrklausel folgte es jedoch uneingeschränkt, ohne in Betracht kommende Besonderheiten Bremerhavens für maßgeblich zu erklären, dem Bundesverfassungsgericht: auch in Bremerhaven sei die 5\%-Klausel verfassungsrechtlich nicht zu rechtfertigen. Es war somit der Staatsgerichtshof, der die Änderung des auf Grund des Volksbegehrens beschlossenen Gesetzes verhinderte.

Allerdings gab er damit dem nichtständigen Ausschuss ein Signal für die beabsichtigte Regelung der Kontralegislatur, wie sie im Zwischenbericht ${ }^{73}$ vorgesehen wurde und jetzt in Art. 73 Abs. 2 LV verankert ist: Das Problem wird auf durch Volksentscheid beschlossene Gesetze beschränkt; die Änderungssperre dafür dauert zwei Jahre, längstens bis zum Ende der Wahlperiode; auch während dieser Sperre sind Änderungen durch Volksentscheid sowie durch die Bürgerschaft mit verfassungsändernder Mehrheit möglich. Aufgrund Volksbegehrens ohne Volksentscheid erlassene Gesetze scheiden daher aus und können ohne rechtliche Grenze geändert werden. Angesichts der oben konstatierten Tendenz,

69 Dazu und zum Folgenden Bgt.-Drs. 17/426 und 17/471; ich habe damals zu der Frage Stellung genommen: D. Schefold, Wieder Fünf-Prozent-Klausel in Bremerhaven?, in: NordÖR 2008, 365 ff.; dort auch weitere Belege.

70 BVerfGE 120, 82.

71 Eine solche präventive Normenkontrolle ist nach Art. 140 LV in Bremen möglich, vgl. A. Rinken, Die Rechtsprechung des Staatsgerichtshofs der Freien Hansestadt Bremen, in: JöR 42 (1994), 325 (328 ff.); zur Problematik sogleich im Folgenden.

72 Urteil v. 14.5.2009 - St 2/08 - unter D I.

73 Bgt.-Drs. 17/594, S. 3, 10, jedoch ohne inhaltliche Begründung. 
Volksentscheide zu vermeiden, bringt diese an sich sachlich vernünftige Regelung wenig.

b) In der Tat blieb die Frage einer die Substanz des neuen Wahlrechts betreffenden Änderung auf der Tagesordnung. Der nichtständige Ausschuss beschäftigte sich nach Erarbeitung der hier dargestellten, am 1. September 2009 in Kraft getretenen Änderung der Verfassung und des Volksentscheid-Gesetzes weiterhin mit seiner Aufgabe der Wahlgesetzgebung und legte schließlich am 23. September 2009 dazu einen umfangreichen Bericht und Antrag vor. ${ }^{74}$ Soweit dieser einen konkreten Gesetzesvorschlag enthielt, fand er mehrheitliche Zustimmung in der Bürgerschaft; ${ }^{75}$ die umfangreiche und viele Einzelheiten, freilich auch die politisch sehr kontroverse Absenkung des Mindestalters für das aktive Wahlrecht betreffende Regelung ließ den sachlichen Gehalt des auf Volksbegehren beschlossenen Gesetzes von 2006 unberührt. Die weiteren Bestrebungen für ein Wahlrecht der EU-Bürger für die Bürgerschaft (Landtag) in Bremen und für ein kommunales Wahlrecht für Drittstaatenangehörige wurden als Aufträge an den Senat zu Bundesratsinitiativen formuliert. All dies kann für das hier behandelte Thema der Neuregelung des Volksentscheids außer Betracht bleiben.

Wohl aber tauchte in den Ausschussberatungen ein Problem auf, das das 2006 beschlossene Gesetz in einem zentralen Punkt betraf. Indem dieses Gesetz (§6), das Modell der starren Landesliste aufgebend, im Rahmen des Verhältniswahlsystems mit Listen dem Wahlberechtigten fünf Stimmen mit der Möglichkeit des Kumulierens und Panaschierens gab, stellte sich die Frage der Verteilung der jedem Wahlvorschlag zustehenden Sitze zunächst auf nach Listenwahl und nach Personenwahl zu besetzende Sitze und dann nach der Reihenfolge der im Weg der Personenwahl und der Listenwahl zu besetzenden Sitze. Das Gesetz beantwortete die erste Frage dahin, dass die Zahl der nach Listenwahl und Personenwahl zu besetzenden Sitze für jede Liste im Verhältnis zur Zahl der für den Wahlvorschlag insgesamt und für seine Bewerber andererseits zu ermitteln sei ( 7 Abs. 5). Es ordnete sodann an, dass erst die nach Listenwahl zu besetzenden Sitze entsprechend der Listenreihenfolge zu besetzen seien, dann die nach Personenwahl zu besetzenden Sitze in der Reihenfolge der erhaltenen Stimmen, soweit die Bewerber nicht schon nach Listenwahl gewählt waren (§ 7 Abs. 6).

Praktische Folge dieser Verteilung ist, dass zunächst die Spitzenkandidaten der Liste bis zur Ausschöpfung der Zahl der nach Listenwahl zu besetzenden Sitze zum Zuge kommen und danach die Bewerber in der Reihenfolge der erhal-

74 Bgt.-Drs. 17/934 vom 23.9.2009, danach auch die im Folgenden referierten Fakten und Argumentationen.

75 Gesetz vom 3.11.2009 (Brem.GBl. S. 443). 
tenen Stimmen, soweit sie nicht schon aufgrund ihres Listenplatzes ihr Mandat erhalten haben. Dies führt dazu, dass mehr Bewerber aufgrund der erhaltenen Stimmen zum Zuge kommen, auch wenn sie weniger Stimmen in der Personenwahl erhalten haben als möglicherweise Spitzenkandidaten auf der Liste: das Element der Personenwahl wird weitest möglich ausgeschöpft, der Einfluss der Listenplatzierung wird reduziert. Die Regelung beeinflusst auch die Listennachfolge bei Erledigung eines Mandats ( $\S 36-36$ b BremWahlG).

Im Ausschuss wurden Bedenken gegen dieses Verteilungssystem geäußert. Auf seine Bitte nahmen die zuständigen Senatoren - für Inneres und für Justiz sowie der Wissenschaftliche Dienst der Bürgerschaft dazu Stellung. Alle befanden - mit unterschiedlicher Dezidiertheit -, dass die gesetzliche Regelung unklar, systemwidrig und daher verfassungsrechtlich bedenklich sei. Alternativ wurde vorgeschlagen, erst die Bewerber mit den meisten Personenstimmen und im übrigen die Bewerber in der Listenreihenfolge für gewählt zu erklären, was das Element der Listenwahl betont - und damit den Kern der 2006 im Volksbegehren geforderten und im Gesetz verwirklichten Reform getroffen hätte. Also ein Fall der Kontralegislatur, wenn auch nicht durch die diesbezügliche Verfassungsvorschrift ausgeschlossen, und mit dem Argument der - freilich bei der Zulassung des Volksbegehrens nicht konstatierten - Verfassungswidrigkeit der Regelung begründet!

Der Ausschuss war sich der Brisanz der Frage bewusst, zögerte mit der Entscheidung und machte sie schließlich von externer Begutachtung, durch die Professoren Hans Meyer (Berlin) und Martin Morlok (Düsseldorf), abhängig. Aber die Gutachten ${ }^{76}$ fielen gegensätzlich aus: Während das Gutachten Morlok darauf abstellte, dass die Personenstimmen Bewerbern zugute kommen könnten, für die sie nicht bestimmt seien, und daher die Verfassungsbedenken, auch im Hinblick auf die Unmittelbarkeit der Wahl, unterstrich, argumentierte das Gutachten Meyer mit der zwangsläufigen Verbindung von Listenstimme auch bei Abgabe einer Personenstimme und bewertete die Frage, ob erst die „Listenbank“ oder die „Personenbank“ für die Sitzvergabe zu berücksichtigen sei, als reine ZweckmäBigkeitsfrage im Rahmen eines personale Elemente berücksichtigenden Verhältniswahlverfahrens, deren Lösung entweder die Abweichung von der Liste - so das geltende Recht - oder die Listenreihenfolge - so der Vorschlag des Innensenators - stärker begünstige; beide Lösungen seien in sich stimmig. Zusätzlich argumentierte das Gutachten mit dem Grundsatz der Organtreue und leitete daraus, freilich ohne sich mit dem damals auf dem Tisch liegenden, erwähnten

76 Beide ebenfalls in dem Ausschuss-Bericht, Bgt.-Drs. 17/934, abgedruckt. 
Vorschlag für Art. 73 Abs. 2 LV auseinander zu setzen, Grenzen der Abänderung des durch Übernahme eines Volksbegehrens erlassenen Gesetzes ab.

Somit war der Ausschuss, auch nach Anhörung der Gutachter und weiterer Diskussion, mit sich widersprechenden Auffassungen konfrontiert. Er war sich der Problematik bewusst, das auf Grund des Volksbegehrens erlassene Gesetz in einem für die Autoren des Volksbegehrens wichtigen Punkt abzuändern, und wollte dies nur im Interesse einer sicheren Rechtsgrundlage, also im Fall durchschlagender verfassungsrechtlicher Bedenken tun. ${ }^{77}$ So lag es nahe, den schon ein Jahr zuvor gegangenen Weg erneut einzuschlagen: der Ausschuss und, auf seinen Antrag, die Bürgerschaft beantragten eine Überprüfung durch den Staatsgerichtshof. Aber diesmal ging es, anders als bei der Idee der Wiedereinführung der 5\%-Klausel in Bremerhaven, um die Frage der Verfassungsmäßigkeit eines geltenden Gesetzes, also nicht um eine präventive, sondern um eine abstrakte Normenkontrolle. ${ }^{78}$

Das Urteil des Staatsgerichtshofs ${ }^{79}$ stellte sich dieser Frage und bejahte die hinreichende Klarheit der Regelung, die Wahrung des Prinzips der Unmittelbarkeit der Wahl und die Folgerichtigkeit der Regelung. Es betonte - insoweit wohl weithin dem Gutachten Meyer folgend - die Notwendigkeit des Vorrangs entweder der Listenbank, oder der Personenbank bei der Mandatszuteilung, und legte dar, dass die Lösung des $§ 7$ Abs. 6 BremWahlG dem im Volksbegehren verfolgten Ziel des Gesetzes, den Einfluss der Wähler auf die Zusammensetzung der Bürgerschaft zu vergrößern, in folgerichtiger Weise diene und auch die Wahlrechtsgleichheit nicht verletze. Der Gesetzgeber sei hinsichtlich der Ausgestaltung des Wahlrechts im Rahmen der verfassungsrechtlichen Grenzen zwar politisch frei - auch hier kein Wort zur Problematik der Organtreue oder Kontralegislatur! -; aber eben deshalb sei die in $\S 7$ Abs. 6 BremWahlG getroffene Lösung nicht zu beanstanden.

Im Ergebnis blieb und bleibt es daher bei der durch das Volksbegehren bewirkten Lösung. Die Bürgerschaft hat zwar zweimal erwogen, in wichtigen Fra-

77 So der Bericht, Bgt.-Drs. 17/934, S. 22 ff.; vgl. auch die in der Anl. 7 dazu abgedruckte Ausschussdiskussion vom 18.8.2009, insb. S. 348 (Abg. Tschöpe), 358 (Abg. Möllenstädt).

78 Art. $140 \mathrm{LV}, \S 24$ StGHG regeln diese zwar nicht separat von der Entscheidung anderer verfassungsrechtlicher Zweifelsfragen, aber der Begriff, geprägt durch Art. 93 I Nr. 2 GG, §§ 76-79 BVerfGG, ist auch für Bremen verwendbar, vgl. Rinken, Rechtsprechung (Fn. 71), S. 329. Allerdings verlangt § 76 I Nr. 1 BVerfGG, dass der Antragsteller das zu prüfende Recht „für nichtig hält“, während § 24 II StGHG Zweifel des Antragstellers genügen lässt.

79 U. v. 8.4.2010 - St 3/09 -, NordÖR 2010, S. 198 ff. 
gen des neuen Rechts dieses zu korrigieren, aber sie hat beide Male diese Entscheidung nicht ohne Rückendeckung durch den Staatsgerichtshof fällen wollen. Beide Male hat der Staatsgerichtshof die Rechtsbedenken gegen die Neuregelung widerlegt. Er hat dabei nicht von Kontralegislatur gesprochen, aber wohl doch eine politische Kultur markiert, die über die - eng begrenzte - Bindungswirkung des Volksentscheids nach Art. 73 Abs. 2 LV hinausweist.

5. Zusammenfassend zeigt die hier referierte Entwicklung, wie langwierig, harzig und oft auch widersprüchlich die Implementation einer im Grundsatz gewollten Volksgesetzgebung ist. Bremen kennt in seiner Nachkriegsverfassung den Volksentscheid seit 1947, aber nach dem damals abgehaltenen doppelten Verfassungsreferendum ist das Institut jahrzehntelang toter Buchstabe geblieben. Die Verfassungsreform von 1994 hat die Anforderungen behutsam gesenkt; aber die Verfassungspraxis von Senat und Staatsgerichtshof, vor allem zum Finanzvorbehalt, hat die Entwicklung weiterhin gebremst, trotz erster Signale für ein Umdenken. Diese haben sich 2006 im Volksbegehren zur Wahlrechtsreform konkretisiert und damit den Anstoß nicht nur dazu, sondern zu einer weiteren Reform der Volksgesetzgebung gegeben. Aber sowohl deren Gehalt, auch mit der möglicherweise problematischen Aufwertung der Stellung der Vertrauenspersonen, wie auch die praktischen Auswirkungen auf die Reform des Wahlrechts zeigen, dass trotz jetzt gegebenen rechtlichen Voraussetzungen der Lernprozess bei der Einbeziehung der Stimmberechtigten noch lang und schwierig sein wird: auf Seiten der Verfassungsorgane, unter denen der Staatsgerichtshof freilich in jüngster Zeit keine Bremsfunktion mehr ausgeübt hat, wie auf Seiten der Bürgerinnen und Bürger. Die Ergänzung der unverzichtbaren und notwendig überwiegenden repräsentativen Demokratie durch Verifikation des Repräsentationsprozesses im Volk bleibt Aufgabe nicht nur rechtlicher, sondern auch politischer Gestaltung. 